\title{
Boundary Layer Effects for the Nonlinear Evolution Equations with the Vanishing Diffusion Limit
}

\author{
Linrui Li $\mathbb{D}^{1}$ and Shu Wang ${ }^{2}$ \\ ${ }^{1}$ Basic Courses Department, Institute of Disaster Prevention, 465 Xueyuan Street, Sanhe, Hebei 065201, China \\ ${ }^{2}$ College of Applied Sciences, Beijing University of Technology, Pingleyuan 100, Chaoyang District, Beijing 100124, China \\ Correspondence should be addressed to Linrui Li; linrui020213@163.com
}

Received 16 January 2018; Accepted 25 March 2018; Published 6 May 2018

Academic Editor: Gabriella Bretti

Copyright ( 2018 Linrui Li and Shu Wang. This is an open access article distributed under the Creative Commons Attribution License, which permits unrestricted use, distribution, and reproduction in any medium, provided the original work is properly cited.

We study an initial-boundary value problem for a nonlinear evolution system with damping and diffusion; our main purpose is to investigate the boundary layer effects when the vanishing diffusion limit $\alpha \rightarrow 0^{+}$, especially for the mixed boundary conditions; we prove that the thickness of layer is of the order $O(\alpha)$. Furthermore, the corresponding convergence rates are also obtained.

\section{Introduction and Main Results}

The main objective of this article is to study the nonlinear evolution equations with damping and diffusion on the domain $[0,1] \times[0, \infty)$ :

$$
\begin{aligned}
& \psi_{t}^{\alpha}=-(k-1) \alpha \psi^{\alpha}-k \alpha \theta_{x}^{\alpha}+\alpha \psi_{x x}^{\alpha}, \\
& \theta_{t}^{\alpha}=-(1-\beta) \theta^{\alpha}+\mu \alpha \psi_{x}^{\alpha}+2 \psi^{\alpha} \theta_{x}^{\alpha}+\beta \theta_{x x}^{\alpha}, \\
& \quad 0<x<1, t>0
\end{aligned}
$$

with initial data

$$
\left(\psi^{\alpha}, \theta^{\alpha}\right)(x, 0)=\left(\psi_{0}, \theta_{0}\right)(x), \quad 0 \leq x \leq 1,
$$

and the mixed boundary conditions, that is, Neumann-Dirichlet boundary conditions

$$
\left(\psi_{x}^{\alpha}, \theta^{\alpha}\right)(0, t)=\left(\psi_{x}^{\alpha}, \theta^{\alpha}\right)(1, t)=(0,0), \quad t \geq 0,
$$

which implies that

$$
\left(\psi_{x t}^{\alpha}, \theta_{t}^{\alpha}\right)(0, t)=\left(\psi_{x t}^{\alpha}, \theta_{t}^{\alpha}\right)(1, t)=(0,0), \quad t \geq 0,
$$

where $k, \alpha, \beta$, and $\mu$ are positive constants with $k>1$ and $0<\beta<1$. The limit problem of the vanishing parameter $\alpha \rightarrow 0^{+}$; we have

$$
\begin{aligned}
& \psi_{t}^{0}=0, \\
& \theta_{t}^{0}=-(1-\beta) \theta^{0}+2 \psi^{0} \theta_{x}^{0}+\beta \theta_{x x}^{0}, \quad 0<x<1, t>0
\end{aligned}
$$

with the initial conditions

$$
\left(\psi^{0}, \theta^{0}\right)(x, 0)=\left(\phi_{1}(x), \phi_{2}(x)\right), \quad 0 \leq x \leq 1,
$$

and the Dirichlet boundary conditions

$$
\theta^{0}(0, t)=\theta^{0}(1, t)=(0,0), \quad t \geq 0,
$$

which implies that

$$
\begin{aligned}
\theta_{t}^{0}(0, t) & =\theta_{t}^{0}(1, t)=0, \\
\left(2 \psi^{0} \theta_{x}^{0}+\beta \theta_{x x}^{0}\right)(0, t) & =\left(2 \psi^{0} \theta_{x}^{0}+\beta \theta_{x x}^{0}\right)(1, t)=0,
\end{aligned}
$$

$t \geq 0$.

We expect to prove that the solution $\left(\psi^{\alpha}, \theta^{\alpha}\right)$ of (1)-(3) converges to the solution $\left(\psi^{0}, \theta^{0}\right)$ of the limit problem (5)-(7), as the diffusion parameter $\alpha \rightarrow 0^{+}$, and get the boundary layer thickness.

The nonlinear interaction between ellipticity and dissipation is involved quite broadly in physical and mechanical systems, such as Rayleigh-Benard problem, superposed fluids, Taylor-Couette instability, dynamic phase transitions, and fluid flow down an inclined plane. [1-3]. As these systems are usually quite complicated, they are far from being well understood. System (1) was originally proposed by Hsieh in [2] to 
investigate the nonlinear interaction between ellipticity and dissipation. We also refer to [1-3] for the physical background of (1). This study is expected to yield insights into physical systems with similar mechanism, such as the Ginzburg-Landau equation and the Kuramoto-Sivashinsky equation $[1,3,4]$; it may also help us, by comparison, to understand better the nonlinear interaction between other instabilities and dissipation.

System (1) has been extensively studied by several authors in different contexts [2, 3, 5-9], such as the well-posedness problems, the nonlinear stability problem, and optimal decay rate of the solutions. However, all the results above need to assume that all parameters are fixed constants. Another interesting problem is the zero diffusion limit: that is, consider the limit problem of solution sequences when one or more of parameters vanishes for the corresponding Cauchy problem or initial-boundary value problem $[5-7,9]$. It should be emphasized that flows often move in bounded domains with constraints from boundaries in real world; in the bounded domains case, the boundary effect requires a careful mathematical analysis. Ruan and Zhu [5] consider the initialboundary value problem (1) with the zero Dirichlet boundary conditions when diffusion parameter $\beta \rightarrow 0^{+}$and they show that the boundary layer thickness $\delta(\beta)$ is of the order $O\left(\beta^{\gamma}\right)$ with $0<\gamma<1 / 2$. Subsequently, Peng et al. [6] consider the initial-boundary value problem (1) with the Dirichlet-Neumann boundary conditions when diffusion parameter $\beta \rightarrow$ $0^{+}$and they show that the boundary layer thickness $\delta(\beta)$ is of the order $O\left(\beta^{\gamma}\right)$ with $0<\gamma<3 / 4$. For the parameter $\alpha \rightarrow$ $0^{+}$, Chen and Zhu in [10] consider the Cauchy problem for (1); Peng in [7] study the initial-boundary value problem for (1) with the zero Dirichlet boundary conditions and obtain the thickness of layer of the order $O\left(\alpha^{1 / 2}\right)$. However, for the asymptotic limit $\alpha \rightarrow 0^{+}$, all these results are restricted to the boundary conditions to the Dirichlet boundary conditions. By [6], which motivates our investigation in this paper, we concentrate our efforts to investigate the boundary layer effect and the convergence rates on the initial-boundary value (1)-(3) with the Dirichlet-Neumann boundary conditions when diffusion parameter $\alpha \rightarrow 0^{+}$, and the solutions of the mixed boundary conditions usually exhibit different behaviors and much rich phenomena comparing with the general boundary conditions. lows.

For later presentation, next we state the notation as fol-

Notations. Throughout this paper, we denote positive constant independent of $\alpha$ by $C$. And the constant $C$ may vary from line to line. $L^{2}=L^{2}([0,1])$ and $L^{\infty}=L^{\infty}([0,1])$ denote the usual Lebesgue space in $[0,1]$ with its norms $\|f\|_{L^{2}([0,1])}=$ $\|f\|=\left(\int_{0}^{1}|f(x)|^{2} d x\right)^{1 / 2}$ and $\|f\|_{L^{\infty}}=\sup _{x \in[0,1]}|f(x)|$. $H^{l}([0,1])$ denotes the usual $l$-th order Sobolev space with its norm $\|f\|_{H^{l}([0,1])}=\|f\|_{l}=\left(\sum_{i=0}^{l}\left\|\partial_{x}^{i} f\right\|^{2}\right)^{1 / 2}$. For simplicity, $\|f(\cdot, t)\|_{L^{2}},\|f(\cdot, t)\|_{L^{\infty}}$ and $\|f\|_{l}$, respectively.

Before we state the main results, let us recall the definition of boundary layer thickness (BL-thickness) in [11] as follows.
Definition 1. A positive function $\delta(\alpha)$ is called a boundary layer thickness for problem (1)-(3) with vanishing diffusion $\alpha$, if $\delta(\alpha) \rightarrow 0$ as $\alpha \rightarrow 0$, and

$$
\begin{aligned}
& \lim _{\alpha \rightarrow 0}\left\|\theta^{\alpha}-\theta^{0}\right\|_{L^{\infty}\left(0, T ; L^{\infty}[0,1]\right)}=0, \\
& \lim _{\alpha \rightarrow 0}\left\|\psi^{\alpha}-\psi^{0}\right\|_{L^{\infty}\left(0, T ; L^{\infty}[\delta, 1-\delta]\right)}=0, \\
& \lim \inf _{\alpha \rightarrow 0}\left\|\psi^{\alpha}-\psi^{0}\right\|_{L^{\infty}\left(0, T ; L^{\infty}[0,1]\right)}>0,
\end{aligned}
$$

where $0<\delta=\delta(\beta)<1,\left(\psi^{\alpha}, \theta^{\alpha}\right)$, and $\left(\psi^{0}, \theta^{0}\right)$ is the solution to problem (1)-(3) and the limit problem (5)-(7), respectively.

Our first purpose is to show that the initial-boundary value problem (1)-(3) admits a unique global smooth solution $\left(\psi^{\alpha}, \theta^{\alpha}\right)$.

Theorem 2. Suppose that the initial data satisfy the conditions: $\left(\psi_{0}, \theta_{0}\right) \in H^{2},\left(\psi_{0}, \theta_{0}\right)(0)=\left(\psi_{0}, \theta_{0}\right)(1)=(0,0)$ and $\left\|\psi_{0}\right\|_{1}+\left\|\theta_{0}\right\|_{1}$ is sufficiently small, then there exists a unique solution $\left(\psi^{\alpha}, \theta^{\alpha}\right)$ to the initial-boundary value problem (1)-(3) satisfying

$$
\begin{aligned}
& \theta^{\alpha} \in L^{\infty}\left(0, T ; H^{2}\right) \cap L^{2}\left(0, T ; H^{2}\right), \\
& \theta_{t}^{\alpha} \in L^{\infty}\left(0, T ; H^{2}\right) \cap L^{2}\left(0, T ; H^{1}\right),
\end{aligned}
$$

where the norms are all uniform in $\alpha$.

Then our second result shows that the initial-boundary value problem (5)-(7) admits a unique global smooth solution $\left(\psi^{0}, \theta^{0}\right)$.

Theorem 3. Suppose that the initial data satisfy the conditions: $\left(\phi_{1}, \phi_{2}\right) \in H^{2}, \phi_{2}(0)=\phi_{2}(1)=0 \leq \varepsilon$, and $\varepsilon$ is sufficiently small, then there exists a unique solution $\left(\psi^{0}, \theta^{0}\right)$ to the initialboundary value problem (5)-(7) satisfying

$$
\begin{aligned}
& \psi^{0} \in L^{\infty}\left(0, T ; H^{2}\right), \\
& \theta^{0} \in L^{\infty}\left(0, T ; H^{2}\right) \cap L^{2}\left(0, T ; H^{2}\right), \\
& \theta_{t}^{0} \in L^{\infty}\left(0, T ; L^{2}\right) \cap L^{2}\left(0, T ; H^{1}\right) .
\end{aligned}
$$

Furthermore, we give the convergence rates and boundary layer thickness.

Theorem 4. Under the assumptions of Theorems 2 and 3, suppose that

$$
\begin{gathered}
\left\|\psi_{0}(x)-\phi_{1}(x)\right\|_{1}^{2} \leq C \alpha, \\
\left\|\theta_{0}(x)-\phi_{2}(x)\right\|_{1}^{2} \leq C \alpha ;
\end{gathered}
$$


then any function $\delta(\alpha)$ satisfying the conditions $\delta(\alpha) \rightarrow 0$ and $\alpha / \delta(\alpha) \rightarrow 0$ as $\alpha \rightarrow 0^{+}$is a BL-thickness such that

$$
\begin{gathered}
\left\|\theta^{\alpha}-\theta^{0}\right\|_{L^{\infty}\left(0, T ; L^{\infty}[0,1]\right)} \leq C \alpha, \\
\left\|\psi^{\alpha}-\psi^{0}\right\|_{L^{\infty}\left(0, T ; L^{\infty}[\delta, 1-\delta]\right)} \leq C \frac{\alpha}{\delta(\alpha)}, \\
\lim \inf _{\alpha \rightarrow 0}\left\|\psi^{\alpha}-\psi^{0}\right\|_{L^{\infty}\left(0, T ; L^{\infty}[0,1]\right)}>0 .
\end{gathered}
$$

Consequently,

$$
\begin{aligned}
\lim _{\alpha \rightarrow 0}\left\|\theta^{\alpha}-\theta^{0}\right\|_{L^{\infty}\left(0, T ; L^{\infty}[0,1]\right)} & =0, \\
\lim _{\alpha \rightarrow 0}\left\|\psi^{\alpha}-\psi^{0}\right\|_{L^{\infty}\left(0, T ; L^{\infty}[\delta, 1-\delta]\right)} & =0 .
\end{aligned}
$$

The plan of this paper is as follows. Section 2 is devoted to the global existence results on the initial-boundary value problem (1)-(3) and the local existence for the limit problem (5)-(7). Section 3 details convergence rates and the BLthickness as the diffusion parameter $\alpha \rightarrow 0^{+}$for the mixed boundary conditions.

\section{Proof of Theorems 2 and 3}

2.1. Proof of Theorem 2. In this subsection, we shall prove Theorem 2 by adapting the elaborate nonlinear energy method. In the following, we devote ourselves to the a priori estimate of solution $\left(\psi^{\alpha}(x, t), \theta^{\alpha}(x, t)\right)$ of (1)-(3) under the a priori assumption

$$
N_{1}(t)=\sup _{0<t<T}\left\|\left(\psi^{\alpha}, \theta^{\alpha}\right)(t)\right\|_{1}^{2} \leq \varepsilon_{1}^{2}
$$

where $\varepsilon_{1}$ is a positive constant satisfying $0<\varepsilon_{1} \leq 1$, independent of $\alpha$.

By Sobolev inequality, we have

$$
\left\|\left(\psi^{\alpha}, \theta^{\alpha}\right)(t)\right\|_{L^{\infty}} \leq \varepsilon_{1} .
$$

\subsection{1. $L^{2}$-Estimates}

Lemma 5. Assume that the conditions in Theorem 2 hold. Then a positive constant $C$ exists independent of $\alpha$, such that

$$
\begin{aligned}
& \frac{1}{2} \int_{0}^{1}\left[\left(\psi^{\alpha}\right)^{2}+\left(\theta^{\alpha}\right)^{2}\right] d x+\int_{0}^{t} \int_{0}^{1}\left[(k-1) \alpha\left(\psi^{\alpha}\right)^{2}\right. \\
& \left.\quad+(1-\beta)\left(\theta^{\alpha}\right)^{2}+\left(\psi_{x}^{\alpha}\right)^{2}+\left(\theta_{x}^{\alpha}\right)^{2}\right] d x d \tau \\
& \quad \leq C
\end{aligned}
$$

Proof. Multiplying the first and the second equations of (1) by $\psi^{\alpha}$ and $\theta^{\alpha}$, respectively, then integrating the resulting equations over $(0,1)$, using integration by parts and the boundary conditions (3), and then adding them, we have

$$
\begin{aligned}
& \frac{1}{2} \frac{d}{d t} \int_{0}^{1}\left[\left(\psi^{\alpha}\right)^{2}+\left(\theta^{\alpha}\right)^{2}\right] d x \\
& \quad+\int_{0}^{1}\left[(k-1) \alpha\left(\psi^{\alpha}\right)^{2}+(1-\beta)\left(\theta^{\alpha}\right)^{2}\right] d x \\
& \quad+\alpha \int_{0}^{1}\left(\psi_{x}^{\alpha}\right)^{2} d x+\beta \int_{0}^{1}\left(\theta_{x}^{\alpha}\right)^{2} d x \\
& =(k+\mu) \alpha \int_{0}^{1} \psi_{x}^{\alpha} \theta^{\alpha} d x-\int_{0}^{1} \psi_{x}^{\alpha}\left(\theta^{\alpha}\right)^{2} d x .
\end{aligned}
$$

Integrating $(20)$ over $(0, t)$, using Cauchy-Schwarz inequality and (18), we obtain for any $p_{1}>0$

$$
\begin{aligned}
& \frac{1}{2} \int_{0}^{1}\left[\left(\psi^{\alpha}\right)^{2}+\left(\theta^{\alpha}\right)^{2}\right] d x \\
& +\int_{0}^{t} \int_{0}^{1}\left[(k-1) \alpha\left(\psi^{\alpha}\right)^{2}+(1-\beta)\left(\theta^{\alpha}\right)^{2}\right] d x d \tau \\
& +\alpha \int_{0}^{t} \int_{0}^{1}\left(\psi_{x}^{\alpha}\right)^{2} d x d \tau+\beta \int_{0}^{1}\left(\theta_{x}^{\alpha}\right)^{2} d x \\
& =\frac{1}{2} \int_{0}^{1}\left[\left(\psi^{\alpha}\right)^{2}+\left(\theta^{\alpha}\right)^{2}\right]_{t=0} d x \\
& +(k+\mu) \alpha \int_{0}^{t} \int_{0}^{1} \psi_{x}^{\alpha} \theta^{\alpha} d x d \tau \\
& \quad-\int_{0}^{t} \int_{0}^{1} \psi_{x}^{\alpha}\left(\theta^{\alpha}\right)^{2} d x d \tau \\
& \quad=\frac{1}{2} \int_{0}^{1}\left[\left(\psi^{\alpha}\right)^{2}+\left(\theta^{\alpha}\right)^{2}\right]_{t=0} d x \\
& +\int_{0}^{t}\left((k+\mu) \alpha+\left\|\theta^{\alpha}(t)\right\|_{L^{\infty}}\right) \int_{0}^{1}\left|\psi_{x}^{\alpha} \theta^{\alpha}\right| d x d \tau \\
& \quad+\frac{1}{2} \int_{0}^{1}\left[\left(\psi_{0}\right)^{2}+\left(\theta_{0}\right)^{2}\right] d x+p_{1} \int_{0}^{t} \int_{0}^{1}\left(\psi_{x}^{\alpha}\right)^{2} d x d \tau \\
& +\left((k+\mu) \alpha+C \varepsilon_{1}\right)^{2} \int_{0}^{t} \int_{0}^{1}\left(\theta^{\alpha}\right)^{2} d x d \tau .
\end{aligned}
$$

If we choose $p_{1}>0$ such that

$$
1-\beta-\frac{\left((k+\mu) \alpha+C \varepsilon_{1}\right)^{2}}{4 p_{1}}>0, \quad \alpha-p_{1}>0,
$$

then we have

$$
\begin{aligned}
& \frac{1}{2} \int_{0}^{1}\left[\left(\psi^{\alpha}\right)^{2}+\left(\theta^{\alpha}\right)^{2}\right] d x \\
& \quad+\int_{0}^{t} \int_{0}^{1}\left[\left(\psi^{\alpha}\right)^{2}+\left(\theta^{\alpha}\right)^{2}+\left(\psi_{x}^{\alpha}\right)^{2}+\left(\theta_{x}^{\alpha}\right)^{2}\right] d x d \tau \\
& \quad \leq C\left\|\left(\psi_{0}, \theta_{0}\right)\right\|^{2} .
\end{aligned}
$$




\subsubsection{High-Order Energy Estimates}

Lemma 6. Assume that the conditions in Theorem 2 hold. Then a positive constant $C$ exists independent of $\alpha$, such that

$$
\begin{aligned}
& \frac{1}{2} \int_{0}^{1}\left[\left(\psi_{x}^{\alpha}\right)^{2}+\left(\theta_{x}^{\alpha}\right)^{2}\right] d x \\
& \quad+\int_{0}^{t} \int_{0}^{1}\left[\left(\psi_{x}^{\alpha}\right)^{2}+\left(\theta_{x}^{\alpha}\right)^{2}+\left(\psi_{x x}^{\alpha}\right)^{2}+\beta\left(\theta_{x x}^{\alpha}\right)^{2}\right] d x d \tau \\
& \quad \leq C
\end{aligned}
$$

Proof. Multiplying the first and the second equations of (1) by $-\psi_{x x}^{\alpha}$ and $-\theta_{x x}^{\alpha}$, respectively, then integrating the resulting equations over $(0,1)$, using integration by parts and the boundary conditions (3), and then adding them, we have

$$
\begin{aligned}
& \frac{1}{2} \frac{d}{d t} \int_{0}^{1}\left[\left(\psi_{x}^{\alpha}\right)^{2}+\left(\theta_{x}^{\alpha}\right)^{2}\right] d x \\
& \quad+\int_{0}^{1}\left[(k-1) \alpha\left(\psi_{x}^{\alpha}\right)^{2}+(1-\beta)\left(\theta_{x}^{\alpha}\right)^{2}\right] d x d \tau \\
& \quad+\alpha \int_{0}^{1}\left(\psi_{x x}^{\alpha}\right)^{2} d x+\beta \int_{0}^{1}\left(\theta_{x x}^{\alpha}\right)^{2} d x \\
& =(k+\mu) \alpha \int_{0}^{1} \psi_{x x}^{\alpha} \theta_{x}^{\alpha} d x+\int_{0}^{1} \psi_{x}^{\alpha}\left(\theta_{x}^{\alpha}\right)^{2} d x .
\end{aligned}
$$

Integrating $(25)$ over $(0, t)$, using Cauchy-Schwarz inequality and (17)-(18), we obtain for any $p_{1}>0$

$$
\begin{aligned}
& \frac{1}{2} \int_{0}^{1}\left[\left(\psi_{x}^{\alpha}\right)^{2}+\left(\theta_{x}^{\alpha}\right)^{2}\right] d x \\
& +\int_{0}^{t} \int_{0}^{1}\left[(k-1) \alpha\left(\psi_{x}^{\alpha}\right)^{2}+(1-\beta)\left(\theta_{x}^{\alpha}\right)^{2}\right] d x \\
& +\alpha \int_{0}^{t} \int_{0}^{1}\left(\psi_{x x}^{\alpha}\right)^{2} d x d \tau+\beta \int_{0}^{t} \int_{0}^{1}\left(\theta_{x x}^{\alpha}\right)^{2} d x d \tau \\
& =\frac{1}{2} \int_{0}^{1}\left[\left(\psi_{x}^{\alpha}\right)^{2}+\left(\theta_{x}^{\alpha}\right)^{2}\right]_{t=0} d x \\
& +(k+\mu) \alpha \int_{0}^{t} \int_{0}^{1} \psi_{x x}^{\alpha} \theta_{x}^{\alpha} d x d \tau \\
& +\int_{0}^{t} \int_{0}^{1} \psi_{x}^{\alpha}\left(\theta_{x}^{\alpha}\right)^{2} d x d \tau \\
& \quad \leq \frac{1}{2} \int_{0}^{1}\left[\left(\psi_{0 x}\right)^{2}+\left(\theta_{0 x}\right)^{2}\right] d x \\
& +p_{1} \int_{0}^{t} \int_{0}^{1}\left(\psi_{x x}^{\alpha}\right)^{2} d x d \tau \\
& +\frac{\left((k+\mu)^{2} \alpha^{2}+C \varepsilon_{1}\right)^{2} \int_{0}^{t} \int_{0}^{1}\left(\theta_{x}^{\beta}\right)^{2} d x d \tau}{4 p_{1}}
\end{aligned}
$$

If we choose $p_{1}>0$ such that

$$
1-\beta-\frac{\left((k+\mu)^{2} \alpha^{2}+C \varepsilon_{1}\right)^{2}}{4 p_{1}}>0, \quad \alpha-p_{1}>0
$$

then we have

$$
\begin{aligned}
& \frac{1}{2} \int_{0}^{1}\left[\left(\psi_{x}^{\alpha}\right)^{2}+\left(\theta_{x}^{\alpha}\right)^{2}\right] d x \\
& +\int_{0}^{t} \int_{0}^{1}\left[\left(\psi_{x}^{\alpha}\right)^{2}+\left(\theta_{x}^{\alpha}\right)^{2}+\alpha\left(\psi_{x x}^{\alpha}\right)^{2}+\beta\left(\theta_{x x}^{\alpha}\right)^{2}\right] d x d \tau \\
& \leq C\left\|\left(\psi_{0}, \theta_{0}\right)\right\|_{1}^{2} .
\end{aligned}
$$

Lemma 7. Let the assumptions of Theorem 2 hold. Then a positive constant $C$ exists independent of $\alpha$, such that

$$
\begin{aligned}
& \int_{0}^{1}\left[\left(\psi_{t}^{\alpha}\right)^{2}+\left(\theta_{t}^{\alpha}\right)^{2}\right] d x \\
& \quad+\int_{0}^{t} \int_{0}^{1}\left[\left(\psi_{x t}^{\alpha}\right)^{2} d x d \tau+\left(\theta_{x t}^{\alpha}\right)^{2}\right] d x d \tau \leq C .
\end{aligned}
$$

Proof. Differentiating (1) with respect to $t$, we get

$$
\begin{aligned}
& \psi_{t t}^{\alpha}=-(k-1) \alpha \psi_{t}^{\alpha}-k \alpha \theta_{x t}^{\alpha}+\alpha \psi_{x x t}^{\alpha}, \\
& \theta_{t t}^{\alpha}=-(1-\beta) \theta_{t}^{\alpha}+\mu \alpha \psi_{x t}^{\alpha}+2\left(\psi^{\alpha} \theta_{x}^{\alpha}\right)_{t}+\beta\left(\theta_{x x t}^{\alpha}\right) .
\end{aligned}
$$

Multiplying the first and the second equations of (1) by $-\psi_{t}^{\alpha}$ and $-\theta_{t}^{\alpha}$, respectively, then integrating the resulting equations over $(0,1)$, using integration by parts and the boundary conditions (3), and then adding them, we have

$$
\begin{aligned}
& \frac{1}{2} \int_{0}^{1}\left[\left(\psi_{t}^{\alpha}\right)^{2}+\left(\theta_{t}^{\alpha}\right)^{2}\right] d x \\
& \quad+\int_{0}^{t} \int_{0}^{1}(k-1) \alpha\left(\psi_{t}^{\alpha}\right)^{2} d x d \tau \\
& \quad+(1-\beta) \int_{0}^{t} \int_{0}^{1}\left(\theta_{t}^{\alpha}\right)^{2} d x d \tau+\alpha \int_{0}^{t} \int_{0}^{1}\left(\psi_{x t}^{\alpha}\right)^{2} d x d \tau \\
& +\beta \int_{0}^{1}\left(\theta_{x t}^{\alpha}\right)^{2} d x d \tau=(k+\mu) \alpha \int_{0}^{t} \int_{0}^{1} \psi_{x t}^{\alpha} \theta_{t}^{\alpha} d x d \tau \\
& \quad+2 \int_{0}^{t} \int_{0}^{1} \psi_{t}^{\alpha} \theta_{x}^{\alpha} \theta_{t}^{\alpha} d x d \tau-\int_{0}^{t} \int_{0}^{1} \psi_{x}^{\alpha}\left(\theta_{t}^{\alpha}\right)^{2} d x d \tau \\
& \quad=\frac{1}{2} \int_{0}^{1}\left[\left(\psi_{t}^{\alpha}\right)^{2}+\left(\theta_{t}^{\alpha}\right)^{2}\right]_{t=0} d x+\sum_{i=1}^{3} J_{i} .
\end{aligned}
$$

We estimate every term as follows:

$$
\begin{aligned}
J_{1} \leq & p_{1} \int_{0}^{t} \int_{0}^{1}\left(\psi_{x t}^{2}\right) d x d \tau \\
& +\frac{(k+\mu)^{2} \alpha^{2}}{4 p_{1}} \int_{0}^{t} \int_{0}^{1}\left(\theta_{t}^{\alpha}\right)^{2} d x d \tau
\end{aligned}
$$


Here $J_{2}, J_{3}$, and $J_{4}$ are estimated as follows:

$$
\begin{aligned}
& J_{2} \leq C \varepsilon_{1} \int_{0}^{t} \int_{0}^{1}\left(\psi_{t}^{\alpha}\right)^{2}+\left(\theta_{t}^{\alpha}\right)^{2} d x d \tau, \\
& J_{3} \leq C \varepsilon_{1} \int_{0}^{t} \int_{0}^{1}\left(\theta_{t}^{\alpha}\right)^{2} d x d \tau .
\end{aligned}
$$

Here we have used Cauchy-Schwarz inequality and Sobolev inequality.

Substituting (32) and (33) into (34), we have from (18) and Lemma 6

$$
\begin{aligned}
& \int_{0}^{1}\left[\left(\psi_{t}^{\alpha}\right)^{2}+\left(\theta_{t}^{\alpha}\right)^{2}\right] d x \\
& \quad+\int_{0}^{t} \int_{0}^{1}\left[\left(\psi_{t}^{\alpha}\right)^{2}+\left(\theta_{t}^{\alpha}\right)^{2}+\left(\psi_{x t}^{\alpha}\right)^{2}+\beta\left(\theta_{x t}^{\alpha}\right)^{2}\right] d x d \tau \\
& \quad \leq C .
\end{aligned}
$$

By the similar method, it is easy to obtain

$$
\int_{0}^{1}\left[\left(\psi_{x x}^{\alpha}\right)^{2}+\beta\left(\theta_{x x}^{\alpha}\right)^{2}\right] d x d \tau \leq C .
$$

Lemma 8. Assume that the conditions in Theorem 2 hold. Then there exists a positive constant $C$ independent of $\alpha$, such that

$$
\begin{array}{r}
\int_{0}^{1}\left(\theta_{x x}^{\alpha}\right)^{2} d x+\int_{0}^{t} \int_{0}^{1}\left(\theta_{x x}^{\alpha}\right)^{2} d x d \tau \\
+\beta \int_{0}^{t} \int_{0}^{1}\left(\theta_{x x x}^{\alpha}\right) d x d \tau \leq C .
\end{array}
$$

Proof. Differentiating the second equation of (1) with respect to $x$, we get

$$
\theta_{x t}^{\alpha}=-(1-\beta) \theta_{x}^{\alpha}+\mu \alpha \psi_{x x}^{\alpha}+2\left(\psi^{\alpha} \theta_{x}^{\alpha}\right)_{x}+\beta \theta_{x x x}^{\alpha} .
$$

Multiplying the equation (37) by $-\theta_{x x x}^{\alpha}$,

$$
\begin{aligned}
& \int_{0}^{1}\left(\theta_{x x}^{\alpha}\right)^{2} d x+\int_{0}^{t} \int_{0}^{1}(1-\beta)\left(\theta_{x x}^{\alpha}\right)^{2} d x d \tau \\
& +\beta \int_{0}^{t} \int_{0}^{1}\left(\theta_{x x x}^{\alpha}\right) d x d \tau=\int_{0}^{1}\left(\theta_{0 x x}\right)^{2} d x \\
& -\mu \int_{0}^{t} \int_{0}^{1}\left[\psi_{t}^{\alpha}+(k-1) \alpha \psi^{\alpha}+k \alpha \theta_{x}^{\alpha}\right] \theta_{x x x}^{\alpha} d x d \tau \\
& -2 \int_{0}^{t} \int_{0}^{1} \psi_{x}^{\alpha} \theta_{x}^{\alpha} \theta_{x x x}^{\alpha} d x d \tau \\
& -2 \int_{0}^{t} \int_{0}^{1} \psi^{\alpha} \theta_{x x}^{\alpha} \theta_{x x x}^{\alpha} d x d \tau=\int_{0}^{1}\left(\theta_{0 x x}\right)^{2} d x \\
& +\mu k \alpha \int_{0}^{t} \int_{0}^{1}\left(\theta_{x x}^{\alpha}\right)^{2} d x d \tau \\
& +\int_{0}^{t} \int_{0}^{1} \mu\left[\psi_{x t}^{\alpha}+(k-1) \alpha \psi_{x}^{\alpha}\right] \theta_{x x}^{\alpha} d x d \tau
\end{aligned}
$$

$$
\begin{aligned}
& +2 \int_{0}^{t} \int_{0}^{1} \psi_{x x}^{\alpha} \theta_{x}^{\alpha} \theta_{x x}^{\alpha} d x d \tau \\
& +3 \int_{0}^{t} \int_{0}^{1} \psi^{\alpha}\left(\theta_{x x}^{\alpha}\right)^{2} d x d \tau=\int_{0}^{1}\left(\theta_{0 x x}\right)^{2} d x \\
& +\mu k \alpha \int_{0}^{t} \int_{0}^{1}\left(\theta_{x x}^{\alpha}\right)^{2} d x d \tau+\sum_{i=4}^{6} J_{i} .
\end{aligned}
$$

We estimate every term as follows:

$$
\begin{aligned}
J_{4} \leq & C \int_{0}^{t} \int_{0}^{1}\left[\left(\psi_{x t}^{\alpha}\right)^{2}+\left(\psi_{x}^{\alpha}\right)^{2}\right] d x d \tau \\
& +p_{1} \int_{0}^{t} \int_{0}^{1}\left(\theta_{x x}^{\alpha}\right)^{2} d x d \tau, \\
J_{5} \leq & C \varepsilon_{1} \int_{0}^{t} \int_{0}^{1}\left(\psi_{x x}^{\alpha}\right)^{2} d x d \tau \\
& +C \varepsilon_{1} \int_{0}^{t} \int_{0}^{1}\left(\theta_{x x}^{\alpha}\right)^{2} d x d \tau, \\
J_{6} \leq & C \varepsilon_{1} \int_{0}^{t} \int_{0}^{1}\left(\theta_{x x}^{\alpha}\right)^{2} d x d \tau .
\end{aligned}
$$

Substituting (39) into (45), we have

$$
\begin{gathered}
\int_{0}^{1}\left(\theta_{x x}^{\alpha}\right)^{2} d x+\int_{0}^{t} \int_{0}^{1}\left(\theta_{x x}^{\alpha}\right)^{2} d x d \tau \\
+\beta \int_{0}^{t} \int_{0}^{1}\left(\theta_{x x x}^{\alpha}\right) d x d \tau \leq C .
\end{gathered}
$$

Now we notice that the priori assumption (18) can be closed. Since, under this priori assumption (18), we deduced that (19), (24), (29), and (36) hold provided that $\varepsilon_{1}$ is sufficiently small. Therefore, assumption (18) is always true provided that $\left\|\psi_{0}, \theta_{0}\right\|_{L^{2}}^{2}$ is sufficiently small.

Lemma 9. Assume that the conditions in Theorem 2 hold. Then a positive constant $C$ exists independent of $\alpha$, such that

$$
\begin{aligned}
& \frac{1}{2} \int_{0}^{1}\left[\left(\psi_{x t}^{\alpha}\right)^{2} d x+\left(\theta_{x t}^{\alpha}\right)^{2}\right] d x \\
& \quad+\int_{0}^{t} \int_{0}^{1}\left[\left(\psi_{x t}^{\alpha}\right)^{2}+\left(\theta_{x t}^{\alpha}\right)^{2}\right] d x d \tau \\
& \quad+\alpha \int_{0}^{t} \int_{0}^{1}\left(\psi_{x x t}^{\alpha}\right)^{2} d x d \tau+\beta \int_{0}^{t} \int_{0}^{1}\left(\theta_{x x t}^{\alpha}\right)^{2} d x d \tau \\
& \leq \text { C. }
\end{aligned}
$$

Proof. Multiplying the first and the second equations of (30) by $-\psi_{x x t}^{\alpha}$ and $-\theta_{x x t}^{\alpha}$, respectively, then integrating the 
6

Discrete Dynamics in Nature and Society

resulting equations over $(0,1)$, using integration by parts and the boundary conditions (3), and then adding them, we have

$$
\begin{aligned}
& \frac{1}{2} \int_{0}^{1}\left[\left(\psi_{x t}^{\alpha}\right)^{2} d x+\left(\theta_{x t}^{\alpha}\right)^{2}\right] d x \\
& +\int_{0}^{t} \int_{0}^{1}\left[(k-1) \alpha\left(\psi_{x t}^{\alpha}\right)^{2}+(1-\beta)\left(\theta_{x t}^{\alpha}\right)^{2}\right] d x d \tau \\
& +\alpha \int_{0}^{t} \int_{0}^{1}\left(\psi_{x x t}^{\alpha}\right) d x d \tau+\beta \int_{0}^{t} \int_{0}^{1}\left(\theta_{x x t}^{\alpha}\right) d x d \tau \\
& =\frac{1}{2} \int_{0}^{1}\left[\left(\psi_{x t}^{\alpha}\right)^{2} d x+\left(\theta_{x t}^{\alpha}\right)^{2}\right]_{t=0} d x \\
& +k \alpha \int_{0}^{t} \int_{0}^{1}\left(\psi_{x x t}^{\alpha}\right) \theta_{x t}^{\alpha} d x d \tau \\
& +\mu \alpha \int_{0}^{t} \int_{0}^{1}\left(\psi_{x x t}^{\alpha}\right) \theta_{x t}^{\alpha} d x d \tau \\
& +2 \int_{0}^{t} \int_{0}^{1}\left(\psi^{\alpha} \theta_{x}^{\alpha}\right)_{t}\left(-\theta_{x x t}^{\alpha}\right) d x d \tau \\
& =\frac{1}{2} \int_{0}^{1}\left[\left(\psi_{x t}^{\alpha}\right)^{2} d x+\left(\theta_{x t}^{\alpha}\right)^{2}\right]_{t=0} d x \\
& +(k+\mu) \alpha \int_{0}^{t} \int_{0}^{1}\left(\psi_{x x t}^{\alpha}\right) \theta_{x t}^{\alpha} d x d \tau \\
& -2 \int_{0}^{t} \int_{0}^{1} \psi_{t}^{\alpha} \theta_{x}^{\alpha} \theta_{x x t}^{\alpha} d x d \tau \\
& -2 \int_{0}^{t} \int_{0}^{1} \psi^{\alpha} \theta_{x t}^{\alpha} \theta_{x x t}^{\alpha} d x d \tau=M \\
& +2 \int_{0}^{t} \int_{0}^{1} \psi_{x t}^{\alpha} \theta_{x}^{\alpha} \theta_{x t}^{\alpha} d x d \tau+2 \int_{0}^{t} \int_{0}^{1} \psi_{t}^{\alpha} \theta_{x x}^{\alpha} \theta_{x t}^{\alpha} d x \\
& +\int_{0}^{t} \int_{0}^{1} \psi_{x}^{\alpha}\left(\theta_{x t}^{\alpha}\right)^{2} d x d \tau=M+\sum_{i=7}^{10} J_{i} .
\end{aligned}
$$

We estimate every term as follows:

$$
\begin{aligned}
& J_{7} \leq p_{1} \int_{0}^{t} \int_{0}^{1}\left(\psi_{x x t}^{\alpha}\right)^{2} d x d \tau \\
&+\frac{[(k+\mu) \alpha]^{2}}{4 p_{1}} \int_{0}^{t} \int_{0}^{1}\left(\theta_{x t}\right)^{2} d x d \tau \\
& J_{8} \leq C \varepsilon_{1} \int_{0}^{t} \int_{0}^{1}\left(\psi_{x t}^{\alpha}\right)^{2} d x d \tau \\
&+C \varepsilon_{1} \int_{0}^{t} \int_{0}^{1}\left(\theta_{x t}^{\alpha}\right)^{2} d x d \tau, \\
& J_{9} \leq 2 \int_{0}^{t} \int_{0}^{1}\left(\psi_{t}^{\alpha} \theta_{x t}^{\alpha}\right)^{2} d x d \tau+\frac{1}{2} \int_{0}^{t} \int_{0}^{1}\left(\theta_{x x}^{\alpha}\right)^{2} d x d \tau \\
& \leq C \int_{0}^{t} \int_{0}^{1}\left[\left(\psi_{t}^{\alpha}\right)^{2}+\left(\psi_{x t}^{\alpha}\right)^{2}\right] d x \int_{0}^{1}\left(\theta_{x t}^{\alpha}\right)^{2} d x d \tau
\end{aligned}
$$

$$
\begin{array}{r}
+\frac{1}{2} \int_{0}^{t} \int_{0}^{1}\left(\theta_{x x}^{\alpha}\right)^{2} d x d \tau, \\
J_{10} \leq C \varepsilon_{1} \int_{0}^{t} \int_{0}^{1}\left(\theta_{x t}^{\alpha}\right)^{2} d x d \tau .
\end{array}
$$

Substituting (43) into (45), we obtain

$$
\begin{aligned}
& \frac{1}{2} \int_{0}^{1}\left[\left(\psi_{x t}^{\alpha}\right)^{2} d x+\left(\theta_{x t}^{\alpha}\right)^{2}\right] d x \\
& \quad+\int_{0}^{t} \int_{0}^{1}\left[\left(\psi_{x t}^{\alpha}\right)^{2}+\left(\theta_{x t}^{\alpha}\right)^{2}\right] d x d \tau \\
& \quad+\alpha \int_{0}^{t} \int_{0}^{1}\left(\psi_{x x t}^{\alpha}\right)^{2} d x d \tau+\beta \int_{0}^{t} \int_{0}^{1}\left(\theta_{x x t}^{\alpha}\right)^{2} d x d \tau \\
& \leq M .
\end{aligned}
$$

By the similar method, we have

$$
\begin{aligned}
& \int_{0}^{1}\left[\left(\psi_{x x x}^{\alpha}\right)^{2} d x+\left(\theta_{x x x}^{\alpha}\right)^{2}\right] d x \\
& \quad+\int_{0}^{t} \int_{0}^{1}\left[\left(\psi_{x x x}^{\alpha}\right)^{2}+\beta\left(\theta_{x x x}^{\alpha}\right)^{2}\right] d x d \tau \leq M .
\end{aligned}
$$

This completes the proof of Lemma 9.

Combination of Lemmas 5-9 and a well-known result on the local existence of the solutions, we can get the global existence of the solutions; this proves Theorem 2 .

2.2. Proof of Theorem 3. Suppose a prior assumption:

$$
A_{2}(T)=\sup _{0<t<T}\left\|\left(\psi_{0}, \theta_{0}\right)(t)\right\|_{3}^{2} \leq \varepsilon_{2},
$$

where $0<\varepsilon_{2} \ll 1$. We obtain the following energy estimates.

Lemma 10. Suppose that the initial data satisfy the conditions: $\phi_{1} \in H^{2}, \phi_{2} \in H^{2}, \phi_{2}(0)=\phi_{2}(1)=0$, and $\left\|\phi_{1}\right\|_{L^{2}}+$ $\left\|\phi_{1}\right\|_{L^{2}} \leq \varepsilon$, and $\varepsilon$ is sufficiently small. Then there exists a unique solution $\left(\psi^{0}, \theta^{0}\right)$ to the initial-boundary value problem (5)-(7) satisfying

$$
\begin{aligned}
& \frac{1}{2} \int_{0}^{1}\left(\theta^{0}\right)^{2} d x+\int_{0}^{t} \int_{0}^{1}\left[\left(\theta^{0}\right)^{2}+\left(\theta_{x}^{0}\right)^{2}\right] d x d \tau \\
& \leq C\left\|\phi_{2}\right\|^{2}, \\
& \int_{0}^{1}\left(\theta_{x}^{0}\right)^{2} d x+\int_{0}^{t} \int_{0}^{1}\left(\theta_{x}^{0}\right)^{2} d x d \tau \\
& +\beta \int_{0}^{t} \int_{0}^{1}\left(\theta_{x x}^{0}\right)^{2} d x d \tau \leq C\left\|\phi_{2}\right\|_{1}^{2}, \\
& \int_{0}^{1}\left[\left(\theta_{t}^{0}\right)^{2}+\left(\theta_{x x}^{0}\right)^{2}\right] d x \\
& +\int_{0}^{t} \int_{0}^{1}\left[\left(\theta_{t}^{0}\right)^{2}+\left(\theta_{x t}^{0}\right)^{2}\right] d x d \tau \\
& \leq C\left(\left\|\phi_{1}\right\|^{2}+\left\|\phi_{2}\right\|_{2}^{2}\right) .
\end{aligned}
$$


Proof. Multiplying the second equation of (5) by $\theta^{0}$, integrating the resulting equation over $(0, t) \times(0,1)$, using integration by part and the boundary conditions (7), and then adding them, we arrive at

$$
\begin{aligned}
& \frac{1}{2} \int_{0}^{1}\left(\theta^{0}\right)^{2} d x+(1-\beta) \int_{0}^{t} \int_{0}^{1}\left(\theta^{0}\right)^{2} d x d \tau \\
& \quad+\beta \int_{0}^{t} \int_{0}^{1}\left(\theta_{x}^{0}\right)^{2} d x d \tau=\frac{1}{2} \int_{0}^{1} \phi_{2}^{2} d x \\
& \quad+2 \int_{0}^{t} \int_{0}^{1} \psi^{0} \theta_{x}^{0} \theta^{2} d x d \tau=C\left\|\phi_{2}\right\|^{2} \\
& \quad+2\left\|\psi^{0}\right\|_{L^{\infty}} \int_{0}^{t} \int_{0}^{1}\left|\theta^{0} \theta_{x}^{0}\right| d x d \tau=C\left\|\phi_{2}\right\|^{2} \\
& \quad+2 \varepsilon_{2} \int_{0}^{t} \int_{0}^{1}\left(\theta^{0}\right)^{2} d x d \tau+C \varepsilon \int_{0}^{t} \int_{0}^{1}\left(\theta_{x}^{0}\right)^{2} d x d \tau .
\end{aligned}
$$

Then we have

$$
\begin{aligned}
& \frac{1}{2} \int_{0}^{1}\left(\theta^{0}\right)^{2} d x+\int_{0}^{t} \int_{0}^{1}\left[\left(\theta^{0}\right)^{2}+\left(\theta_{x}^{0}\right)^{2}\right] d x d \tau \\
& \quad \leq C\left\|\phi_{2}\right\|^{2} .
\end{aligned}
$$

Differentiating the second equation of (5) with respect to $x$, we get

$$
\theta_{x t}^{0}=-(1-\beta) \theta_{x}^{0}+2\left(\psi^{0} \theta_{x}^{0}\right)_{x}+\beta \theta_{x x x}^{0} .
$$

Multiplying (52) by $\theta_{x}^{0}$, using integration by part and the boundary conditions, we obtain

$$
\begin{aligned}
& \frac{1}{2} \int_{0}^{1}\left(\theta_{x}^{0}\right)^{2} d x+(1-\beta) \int_{0}^{t} \int_{0}^{1}\left(\theta_{x}^{0}\right)^{2} d x d \tau \\
& \quad+\beta \int_{0}^{t} \int_{0}^{1}\left(\theta_{x x}^{\alpha}\right)^{2} d x d \tau=\left.\frac{1}{2} \int_{0}^{1}\left(\theta_{x}^{0}\right)^{2}\right|_{t=0} d x \\
& \quad+2 \int_{0}^{t} \int_{0}^{1} \psi_{x}^{0}\left(\theta_{x}^{0}\right)^{2} d x d \tau \\
& \quad+2 \int_{0}^{t} \int_{0}^{1}\left(\psi^{0} \theta_{x x}^{0}\right) \theta_{x}^{0} d x d \tau \leq \frac{1}{2} \int_{0}^{1}\left(\phi_{2 x}(x)\right) d x \\
& \quad+2\left\|\psi_{x}^{0}\right\|_{L^{\infty}} \int_{0}^{t} \int_{0}^{1}\left(\theta_{x}^{0}\right)^{2} d x d \tau+2\left\|\psi^{0}\right\|_{L^{\infty}} \\
& \quad+\left(\int_{0}^{t} \int_{0}^{1}\left(\theta_{x x}^{0}\right)^{2} d x d \tau+\int_{0}^{t} \int_{0}^{1}\left(\theta_{x}^{0}\right)^{2} d x d \tau\right) \\
& \quad \leq C\left\|_{2}\right\|_{1}^{2} .
\end{aligned}
$$

Therefore, we have

$$
\begin{aligned}
& \int_{0}^{1}\left(\theta_{x}^{0}\right)^{2} d x+\int_{0}^{t} \int_{0}^{1}\left(\theta_{x}^{0}\right)^{2} d x d \tau \\
& \quad+\beta \int_{0}^{t} \int_{0}^{1}\left(\theta_{x x}^{0}\right)^{2} d x d \tau \leq C\left\|\phi_{2}\right\|_{1}^{2} .
\end{aligned}
$$

Moreover, if we differentiate the second equation of (5) with respect to $t$, we have

$$
\theta_{t t}^{0}=-(1-\beta) \theta_{t}^{0}+2\left(\psi^{0} \theta_{x}^{0}\right)_{t}+\beta\left(\theta_{x x}^{0}\right)_{t}
$$

and then multiplying (55) by $\theta_{t}^{0}$ and integrating the resulting equation over $(0, t) \times(0,1)$, using integration by part and the boundary conditions (7), we obtain

$$
\begin{aligned}
& \frac{1}{2} \int_{0}^{1}\left(\theta_{t}^{0}\right)^{2} d x+(1-\beta) \int_{0}^{t} \int_{0}^{1}\left(\theta_{t}^{0}\right)^{2} d x d \tau \\
& \quad+\beta \int_{0}^{t} \int_{0}^{1}\left(\theta_{x t}^{0}\right)^{2} d x d \tau=\frac{1}{2} \int_{0}^{1} \theta_{0 t} d x \\
& \quad+2 \int_{0}^{t} \int_{0}^{1}\left(\psi^{0} \theta_{x}^{0}\right)_{t} \theta_{t}^{0} \leq \frac{1}{2}\left\|\phi_{2 t}\right\|^{2} \\
& \quad+2 \int_{0}^{t} \int_{0}^{1}\left(\psi_{t}^{0} \theta_{x}^{0}+\psi^{0} \theta_{x t}^{0}\right) d x d \tau=\frac{1}{2} C \\
& \quad+2\left\|\psi_{t}^{0}\right\|_{L^{\infty}} \int_{0}^{t} \int_{0}^{1}\left(\theta_{x}^{0}\right)^{2} d x d \tau \\
& \quad+2\left\|\psi^{0}\right\|_{L^{\infty}} \int_{0}^{t} \int_{0}^{1}\left(\theta_{x t}^{0}\right)^{2} d x d \tau ;
\end{aligned}
$$

then we have

$$
\int_{0}^{1}\left(\theta_{t}^{0}\right)^{2} d x \leq C
$$

Utilizing the above estimates and the similar produces, by the assumption of $\varepsilon$, we have

$$
\begin{aligned}
& \frac{1}{2} \int_{0}^{1}\left(\theta_{t}^{0}\right)^{2} d x+(1-\beta) \int_{0}^{t} \int_{0}^{1}\left(\theta_{t}^{0}\right)^{2} d x d \tau \\
& \quad+\beta \int_{0}^{t} \int_{0}^{1}\left(\theta_{x t}^{0}\right)^{2} d x d \tau \leq C\left(\left\|\phi_{1}\right\|_{2}^{2}+\left\|\phi_{2}\right\|_{2}^{2}\right) .
\end{aligned}
$$

Therefore,

$$
\begin{aligned}
& \int_{0}^{1}\left(\theta_{x x}^{0}\right)^{2} d x \leq C \int_{0}^{1}\left[\left(\theta_{t}^{0}\right)^{2}+\left(\theta^{0}\right)^{2}+\left(\psi^{0} \theta_{x}^{0}\right)^{2}\right] d x \\
& \quad \leq C \int_{0}^{1}\left[\left(\theta_{t}^{0}\right)^{2}+\left(\theta^{0}\right)^{2}+\left(\psi^{0}\right)^{2}+\left(\theta_{x}^{0}\right)^{2}\right] d x \\
& \quad \leq C\left(\left\|\phi_{1}\right\|_{2}^{2}+\left\|\phi_{2}\right\|_{2}^{2}\right) .
\end{aligned}
$$

Here we have used (47), (48), (58), and (59). The proof of Lemma 10 is finished.

Finally, based on Lemma 10, we can prove Theorem 3.

\section{Convergence Rates and BL-Thickness}

In this section, we turn another interesting problem, which is concerned with $L^{2}$ convergence rates of the vanishing diffusion viscosity parameter $\alpha \rightarrow 0^{+}$and the BL-thickness. That is, we will give the proof of Theorem 4 , and it suffices to show the following two lemmas. 
8

Discrete Dynamics in Nature and Society

Lemma 11. Assume that the conditions in Theorem 4 hold. Then we have

$$
\begin{aligned}
& \int_{0}^{1}\left[\left(\psi^{\alpha}-\psi^{0}\right)^{2}+\left(\theta^{\alpha}-\theta^{0}\right)^{2}\right] d x \\
& \quad+\int_{0}^{t} \int_{0}^{1}\left[\alpha\left(\psi^{\alpha}-\psi^{0}\right)^{2}+\left(\theta^{\alpha}-\theta^{0}\right)^{2}\right. \\
& \left.\quad+\left(\theta^{\alpha}-\theta^{0}\right)_{x}^{2}\right] d x d \tau \leq C \alpha, \\
& \int_{0}^{1}\left(\theta^{\alpha}-\theta^{0}\right)_{x}^{2} d x+\int_{0}^{t} \int_{0}^{1}\left(\theta^{\alpha}-\theta^{0}\right)_{t}^{2} d x d \tau \leq C \alpha,
\end{aligned}
$$

where $C$ is a positive constant, independent of $\alpha$.

Proof. Set $u^{\alpha}=\psi^{\alpha}-\psi^{0}, v^{\alpha}=\theta^{\alpha}-\theta^{0}$; then we have

$$
\begin{aligned}
& u_{t}^{\alpha}=-(k-1) \alpha u^{\alpha}-(k-1) \alpha \psi^{0}-k \alpha \theta_{x}^{\alpha}+\alpha \psi_{x x}^{\alpha}, \\
& v_{t}^{\alpha}=-(1-\beta) v^{\alpha}+\mu \alpha \psi_{x}^{\alpha}+2 \psi^{\alpha} v_{x}^{\alpha}+2 u^{\alpha} \theta_{x}^{0}+\beta v_{x x}^{\alpha}
\end{aligned}
$$

with initial data

$$
\begin{array}{r}
\left(u^{\alpha}, v^{\alpha}\right)(x, 0)=\left(\psi_{0}(x)-\phi_{1}(x), \theta_{0}(x)-\phi_{2}(x)\right), \\
0 \leq x \leq 1,
\end{array}
$$

and boundary condition

$$
v^{\alpha}(0, t)=v^{\alpha}(1, t)=0,
$$

which implies that

$$
v_{t}^{\alpha}(0, t)=v_{t}^{\alpha}(1, t)=0, \quad t \geq 0 .
$$

Multiplying the first equation of (61) by $u_{\alpha}$ and the second equation of (61) by $v_{\alpha}$, respectively, and then adding them, we have

$$
\begin{aligned}
& \frac{1}{2} \int_{0}^{1}\left[\left(u^{\alpha}\right)^{2}+\left(v^{\alpha}\right)^{2}\right] d x+\int_{0}^{t} \int_{0}^{1}\left[(k-1) \alpha\left(u^{\alpha}\right)^{2}\right. \\
& \left.+(1-\beta)\left(v^{\alpha}\right)^{2}+\beta\left(v_{x}^{\alpha}\right)^{2}\right] d x d \tau=\frac{1}{2} \\
& \cdot \int_{0}^{1}\left[\left(u^{\alpha}\right)^{2}+\left(v^{\alpha}\right)^{2}\right]_{t=0} d x-(k-1) \\
& \cdot \alpha \int_{0}^{t} \int_{0}^{1}\left(u^{\alpha}\right) \psi^{0} d x d \tau-k \alpha \int_{0}^{t} \int_{0}^{1} \theta_{x}^{\alpha} u^{\alpha} d x d \tau \\
& +\mu \alpha \int_{0}^{t} \int_{0}^{1} \psi_{x}^{\alpha} v^{\alpha} d x d \tau+2 \int_{0}^{t} \int_{0}^{1} \psi^{\alpha} v_{x}^{\alpha} v^{\alpha} d x d \tau \\
& +2 \int_{0}^{t} \int_{0}^{1} u^{\alpha} \theta_{x}^{0} v^{\alpha} d x d \tau+\alpha \int_{0}^{t} \int_{0}^{1} \psi_{x x}^{\alpha} u^{\alpha} d x d \tau=\frac{1}{2} \\
& \cdot \int_{0}^{1}\left[\left|\psi_{0}(x)-\phi_{1}(x)\right|^{2}+\left|\theta_{0}(x)-\phi_{2}(x)\right|^{2}\right] d x \\
& \quad-(k-1) \alpha \int_{0}^{t} \int_{0}^{1} \psi^{0} u^{\alpha} d x d \tau \\
& -k \alpha \int_{0}^{t} \int_{0}^{1} \theta_{x}^{\alpha} u^{\alpha} d x d \tau+\mu \alpha \int_{0}^{t} \int_{0}^{1} \psi_{x}^{\alpha} v^{\alpha} d x d \tau
\end{aligned}
$$

$$
\begin{aligned}
& -\int_{0}^{t} \int_{0}^{1} \psi_{x}^{\alpha}\left(v^{\alpha}\right)^{2} d x d \tau=\frac{1}{2} \int_{0}^{1}\left[\left|\psi_{0}(x)-\phi_{1}(x)\right|^{2}\right. \\
& \left.+\left|\theta_{0}(x)-\phi_{2}(x)\right|^{2}\right] d x+\sum_{i=1}^{6} M_{i} .
\end{aligned}
$$

Then we estimate every term $M_{1}-M_{6}$ as follows:

$$
\begin{aligned}
& M_{1} \leq \frac{(k-1) \alpha}{2} \int_{0}^{t} \int_{0}^{1}\left(\psi^{0}\right)^{2} d x d \tau \\
& +\frac{(k-1) \alpha}{2} \int_{0}^{t} \int_{0}^{1}\left(u^{\alpha}\right)^{2} d x d \tau, \\
& M_{2} \leq k \int_{0}^{t} \int_{0}^{1}\left(\theta_{x}^{\alpha}\right)^{2} d x d \tau \\
& +\frac{(k-1) \alpha}{2 k} \int_{0}^{t} \int_{0}^{1}\left(u^{\alpha}\right)^{2} d x d \tau, \\
& M_{3} \leq \frac{\mu^{2} \alpha^{2}}{4 \lambda} \int_{0}^{t} \int_{0}^{1}\left(\psi_{x}^{\alpha}\right)^{2} d x d \tau \\
& +C \lambda \int_{0}^{t} \int_{0}^{1}\left(v^{\alpha}\right)^{2} d x d \tau, \\
& M_{4}+M_{5}=2 \int_{0}^{t} \int_{0}^{1} \psi v_{x}^{\alpha} v^{\alpha} d x d \tau \\
& +2 \int_{0}^{t} \int_{0}^{1} u^{\alpha} \theta_{x}^{0} v^{\alpha} d x d \tau \\
& \leq C \varepsilon \int_{0}^{t} \int_{0}^{1}\left(v_{x}^{\alpha}\right)^{2} d x d \tau \\
& +C \varepsilon \int_{0}^{t} \int_{0}^{1}\left(v^{\alpha}\right)^{2} d x d \tau \\
& +C \delta \int_{0}^{t} \int_{0}^{1}\left(u^{\alpha}\right)^{2} d x d \tau \\
& +C \delta \int_{0}^{t} \int_{0}^{1}\left(v^{\alpha}\right)^{2} d x d \tau \\
& M_{6} \leq \frac{\alpha^{2}}{4 \lambda} \int\left(\psi_{x x}^{\alpha}\right)^{2} d x d \tau \\
& +\lambda \int_{0}^{t} \int_{0}^{1}\left(u^{\alpha}\right)^{2} d x d \tau .
\end{aligned}
$$

Putting (66) into (65), we obtain

$$
\begin{aligned}
& \int_{0}^{1}\left[\left(u^{\alpha}\right)^{2}+\left(v^{\alpha}\right)^{2}\right] d x \\
& \quad+\int_{0}^{t} \int_{0}^{1}\left[\alpha\left(u^{\alpha}\right)^{2}+\left(v^{\alpha}\right)^{2}+\left(v_{x}^{\alpha}\right)^{2}\right] d x d \tau \\
& \leq C \alpha+(\lambda+C \delta) \int_{0}^{t} \int_{0}^{1}\left(u^{\alpha}\right)^{2} d x d \tau .
\end{aligned}
$$


By Gronwall's inequality, we have

$$
\begin{aligned}
& \int_{0}^{1}\left[\left(u^{\alpha}\right)^{2}+\left(u^{\alpha}\right)^{2}\right] d x \\
& \quad+\int_{0}^{t} \int_{0}^{1}\left[\alpha\left(u^{\alpha}\right)^{2}+\left(v^{\alpha}\right)^{2}+\left(v_{x}^{\alpha}\right)^{2}\right] d x d \tau \leq C \alpha .
\end{aligned}
$$

Multiplying the second equation of (61) by $v_{t}^{\alpha}$, we have

$$
\begin{aligned}
& \frac{1}{2}(1-\beta) \int_{0}^{1}\left(v^{\alpha}\right)^{2} d x+\frac{1}{2} \beta \int_{0}^{1}\left(v_{x}^{\alpha}\right)^{2} d x \\
& \quad+\int_{0}^{t} \int_{0}^{1}\left(v_{t}^{\alpha}\right)^{2} d x d \tau \\
& \quad=\frac{1}{2} \int_{0}^{1}\left[(1-\beta)\left(v^{\alpha}\right)^{2}+\beta\left(v_{x}^{\alpha}\right)^{2}\right]_{t=0} d x \\
& +\int_{0}^{t} \int_{0}^{1} \mu \alpha \psi_{x}^{\alpha} v_{t}^{\alpha} d x d \tau+2 \int_{0}^{t} \int_{0}^{1} \psi^{\alpha} v_{x}^{\alpha} v_{t}^{\alpha} d x d \tau \\
& \quad+2 \int_{0}^{t} \int_{0}^{1} u^{\alpha} \theta_{x}^{0} v_{t}^{\alpha} d x d \tau=\sum_{i=7}^{10} M_{i} .
\end{aligned}
$$$$
\left.+\beta\left(\theta_{0}(x)-\phi_{2}(x)\right)_{x}^{2}\right] d x \leq C \| \theta_{0}
$$$$
-\phi_{2}(x) \|_{1}^{2} \leq C \alpha,
$$$$
M_{8} \leq \frac{\mu^{2} \alpha^{2}}{2} \int_{0}^{t} \int_{0}^{1}\left[\left(\phi_{x}^{\alpha}\right)^{2}+\frac{1}{2}\left(v_{t}^{\alpha}\right)^{2}\right] d x d \tau \leq C \alpha+\frac{1}{2}
$$$$
\int_{0}^{t} \int_{0}^{1}\left(v_{t}^{\alpha}\right)^{2} d x d \tau
$$$$
M_{9} \leq C \varepsilon \int_{0}^{t} \int_{0}^{1}\left(v_{x}^{\alpha}\right)^{2} d x d \tau+C \varepsilon \int_{0}^{t} \int_{0}^{1}\left(v_{t}^{\alpha}\right)^{2} d x d \tau,
$$$$
M_{10} \leq C \delta \int_{0}^{t} \int_{0}^{1}\left(u^{\alpha}\right)^{2} d x d \tau+C \delta\left(v_{t}^{\alpha}\right)^{2} d x d \tau
$$$$
\leq C \delta \alpha+C \delta \int_{0}^{t} \int_{0}^{1}\left(v_{t}^{\alpha}\right)^{2} d x d \tau
$$

Collecting (70)-(73) and putting into (69), we have

$$
\frac{1}{2} \int_{0}^{1}\left[\left(v^{\alpha}\right)^{2}+\left(v_{x}^{\alpha}\right)^{2}\right] d x+\int_{0}^{t} \int_{0}^{1}\left(v_{t}^{\alpha}\right)^{2} d x d \tau \leq C \alpha .
$$

Utilizing the similar step, multiplying the second equation of (61) by $v_{t}^{\alpha}$, then integrating the resulting equation over $(0, t) \times(0,1)$, and then estimating every term, finally we obtain

$$
\int_{0}^{1}\left[\left(v^{\alpha}\right)^{2}+\left(v_{x}^{\alpha}\right)^{2}\right] d x+\int_{0}^{t} \int_{0}^{1}\left(v_{t}^{\alpha}\right)^{2} d x d \tau \leq C \alpha .
$$

The following lemma will be devoted to the boundary layer thickness.

Lemma 12. Assume that the conditions in Theorem 2 hold. Then we have

$$
\delta \int_{\delta}^{1-\delta}\left|\psi_{x}^{\alpha}-\psi_{x}^{0}\right| d x \leq C \alpha
$$

Proof. Differentiating (62) with respect to $x$, we get

$$
u_{x t}^{\alpha}=-(k-1) \alpha u_{x}^{\alpha}-(k-1) \alpha \psi_{x}^{0}-k \alpha \theta_{x x}^{\alpha}+\alpha \psi_{x x x}^{\alpha} .
$$

Let $u_{x}^{\alpha}=\omega$; then we have

$$
\omega_{t}=-(k-1) \alpha \omega-(k-1) \alpha \psi_{x}^{0}-k \alpha \theta_{x x}^{\alpha}+\alpha \psi_{x x x}^{\alpha} .
$$

To construct a cut-off function as in [12], we define $\varphi_{\eta}$, for $\eta \in(0,1)$, and $\xi_{\delta}$ for $\delta \in(0,1 / 2)$, by

$$
\begin{aligned}
& \varphi_{\eta}(s)= \sqrt{s^{2}+\eta^{2}}, \\
& \xi_{\delta}= \begin{cases}x, & 0 \leq x \leq \delta, \\
\delta, & \delta \leq x \leq 1-\delta, \\
1-x, & 1-\delta \leq x \leq 1 .\end{cases}
\end{aligned}
$$

It is easy to check that $\varphi_{\eta}$ satisfies

$$
\begin{aligned}
|s| & \leq\left|\varphi_{\eta}(s)\right| \leq|s|+1, \\
\left|\varphi_{\eta}^{\prime}(s)\right| & \leq 1, \\
0 & \leq s \varphi_{\eta}^{\prime}(s) \leq \varphi_{\eta}(s), \\
\varphi_{\eta}^{\prime \prime}(s) & \geq 0, \\
s^{2} \varphi_{\eta}^{\prime \prime}(s) & \geq 0 .
\end{aligned}
$$

$\xi_{\delta}$ satisfies $0 \leq \xi_{\delta} \leq \delta, \xi_{\delta}(1)=\xi_{\delta}(0)=0$, multiplying (78) by $\varphi_{\eta}^{\prime}(\omega) \xi_{\delta}$ and then integrating over

$$
\begin{gathered}
\int_{0}^{t} \int_{0}^{1} \varphi_{\eta}^{\prime}(\omega) \xi_{\delta} d x-\varepsilon \int_{0}^{1} \xi_{\delta} d x \\
=-(k-1) \alpha \int_{0}^{t} \int_{0}^{1} \omega \varphi_{\eta}^{\prime}(\omega) \xi_{\delta} d x d \tau \\
-(k-1) \alpha \int_{0}^{t} \int_{0}^{1} \psi_{x}^{0} \varphi_{\eta}^{\prime}(\omega) \xi_{\delta} d x d \tau \\
-k \alpha \int_{0}^{t} \int_{0}^{1} \theta_{x x}^{\alpha} \varphi_{\eta}^{\prime}(\omega) \xi_{\delta} d x d \tau \\
+\alpha \int_{0}^{t} \int_{0}^{1} \psi_{x x x}^{\alpha} \varphi_{\varepsilon}^{\prime}(\omega) \xi_{\delta} d x d \tau=\sum_{i=1}^{4} M_{i} .
\end{gathered}
$$


By the fact that $0 \leq s \varphi_{\eta}^{\prime}(s) \leq \varphi_{\eta}(s)$, we have

$$
M_{1} \leq \int_{0}^{t} \int_{0}^{1} \varphi_{\varepsilon}(\omega) \xi_{\delta} d x d \tau
$$

By $\varphi_{\eta}^{\prime}(\omega) \leq 1,0 \leq \xi_{\delta} \leq \delta$, we obtain

$$
\begin{aligned}
& M_{2} \leq C \delta\left[\int_{0}^{t} \int_{0}^{1}\left(\varphi_{x}^{0}\right)^{2} d x d \tau\right]^{1 / 2} \leq C \delta \alpha \\
& M_{3} \leq C \alpha \delta\left[\int_{0}^{t} \int_{0}^{1}\left(\theta_{x x}^{\alpha}\right)^{2} d x d \tau\right]^{1 / 2} \leq C \delta \alpha, \\
& M_{4} \leq C \alpha \delta \int_{0}^{t} \int_{0}^{1}\left(\theta_{x x x}^{\alpha}\right)^{2} d x d \tau \leq C \alpha
\end{aligned}
$$

Combining (82) and (83), we notice that

$$
\begin{gathered}
\varepsilon \int_{0}^{1} \xi_{\delta} d x \leq \varepsilon \delta \\
\int_{0}^{t} \int_{0}^{1} \varphi_{\eta}^{\prime}(\omega) \xi_{\delta} d x d \tau \leq C \int_{0}^{t} \int_{0}^{1} \varphi_{\eta}(\omega) \xi_{\delta} d x d \tau \\
+C \alpha \delta+\epsilon \delta .
\end{gathered}
$$

By Gronwall's inequality, we have

$$
\int_{0}^{1} \varphi_{\eta}(\omega) \xi_{\delta} d x \leq C(\varepsilon \delta+\alpha \delta) .
$$

By the definition of $\xi_{\delta},|z| \leq \varphi_{\eta}(\omega)$, we have

$$
\int_{\delta}^{1-\delta}|\omega| d x \leq C(\varepsilon \delta+\alpha \delta)<C(\varepsilon+\alpha)
$$

Letting $\varepsilon \rightarrow 0$,

$$
\int_{\delta}^{1-\delta}|\omega| d x \leq C \alpha
$$

Because $W^{1,1}([\delta, 1-\delta]) \hookrightarrow L^{\infty}([\delta, 1-\delta])$, it follows that

$$
\begin{aligned}
\left\|\psi^{\alpha}-\psi^{0}\right\|_{L^{\infty}([\delta, 1-\delta])} \leq & \int_{0}^{1}\left|\psi^{\alpha}-\psi^{0}\right| d x \\
& +\int_{\delta}^{1-\delta}\left|\psi_{x}^{\alpha}-\psi_{x}^{0}\right| d x \leq C \alpha .
\end{aligned}
$$

As in $[5,6,13]$, we observe that inequality (11) holds. This completes the proof of Theorem 4.

\section{Conflicts of Interest}

The authors declare that they have no conflicts of interest.

\section{Authors' Contributions}

The paper was finished in collaboration among all authors. Linrui Li found the motivation of this paper and suggested the outline of the proofs. Shu Wang provided good ideas and gave some valuable suggestions for completing the paper. All authors of the manuscript have read and agreed to its content and are accountable for all aspects of the accuracy and integrity of the manuscript.

\section{Acknowledgments}

This research was supported by the Science and Technology Planning Project of Hebei Province (Grant no. 16275432) and the National Science Foundation of China (Grant no. 11371042). This research was done during Dr. Linrui Li's visit to College of Applied Sciences in Beijing University of Technology. She would like to thank Professor Shu Wang for his many useful suggestions and stimulating discussions on this work.

\section{References}

[1] L. R. Keefe, "Dynamics of perturbed wavetrain solutions to the Ginzburg-Landau equation," Studies in Applied Mathematics, vol. 73, no. 2, pp. 91-153, 1985.

[2] D. Y. Hsieh, "On partial differential equations related to Lorenz system," Journal of Mathematical Physics, vol. 28, no. 7, pp. 15891597, 1987.

[3] Y. Kuramoto and T. Tsuzuki, "On the formation of dissipative structures in reaction-diffusion systems," Progress of Theoretical Physics, vol. 54, no. 3, pp. 687-699, 1975.

[4] G. I. Sivashinsky, "Nonlinear analysis of hydrodynamic instability in laminar flames. I. Derivation of basic equations," Acta Astronautica. Journal of the International Academy of Astronautics, vol. 4, no. 11-12, pp. 1177-1206, 1977.

[5] L. Ruan and C. Zhu, "Boundary layer for nonlinear evolution equations with damping and diffusion," Discrete and Continuous Dynamical Systems - Series A, vol. 32, no. 1, pp. 331-352, 2012.

[6] H. Peng, L. Ruan, and J. Xiang, "A note on boundary layer of a nonlinear evolution system with damping and diffusions," Journal of Mathematical Analysis and Applications, vol. 426, no. 2, pp. 1099-1129, 2015.

[7] Y. Peng, "Boundary layer and vanishing diffusion limit for nonlinear evolution equations," Acta Mathematica Scientia B, vol. 34, no. 4, pp. 1271-1286, 2014.

[8] C. Zhu and Z. Wang, "Decay rates of solutions to dissipative nonlinear evolution equations with ellipticity," Zeitschrift für angewandte Mathematik und Physik ZAMP, vol. 55, no. 6, pp. 994-1014, 2004.

[9] R. Duan and C. Zhu, "Asymptotics of dissipative nonlinear evolution equations with ellipticity: different end states," Journal of Mathematical Analysis and Applications, vol. 303, no. 1, pp. 15-35, 2005.

[10] K. Chen and C. Zhu, "The zero diffusion limit for nonlinear hyperbolic system with damping and diffusion," Journal of Hyperbolic Differential Equations, vol. 5, no. 4, pp. 767-783, 2008.

[11] H. Frid and V. Shelukhin, "Boundary layers in parabolic perturbations of scalar conservation laws," Zeitschrift für angewandte Mathematik und Physik ZAMP, vol. 55, no. 3, pp. 420-434, 2004. 
[12] W. Zhou, X. Qin, and X. Wei, "An estimate on the thickness of boundary layer for nonlinear evolution equations with damping and diffusion," Journal of Inequalities and Applications, Paper No. 249, 7 pages, 2016.

[13] H. Frid and V. Shelukhin, "Boundary layers for the NavierStokes equations of compressible fluids," Communications in Mathematical Physics, vol. 208, no. 2, pp. 309-330, 1999. 


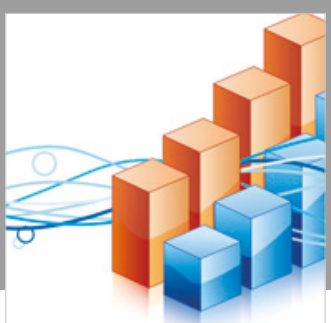

Advances in

Operations Research

\section{-n-m}
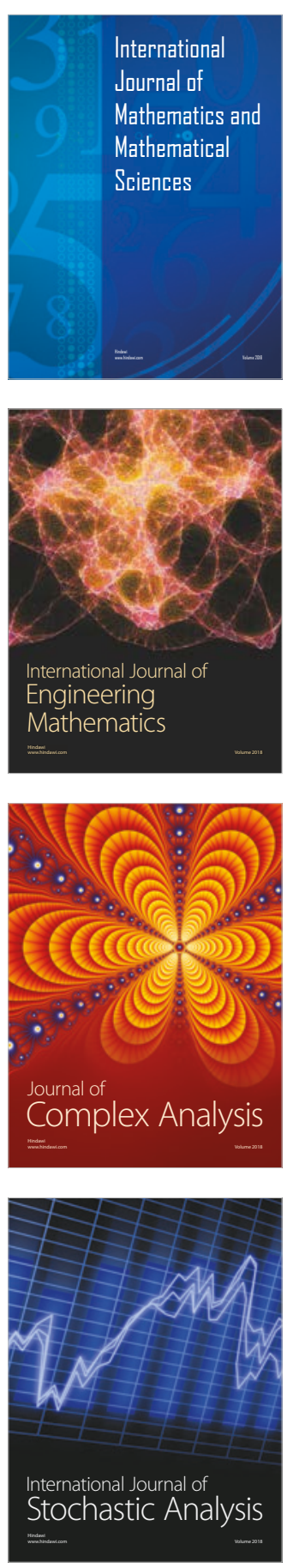
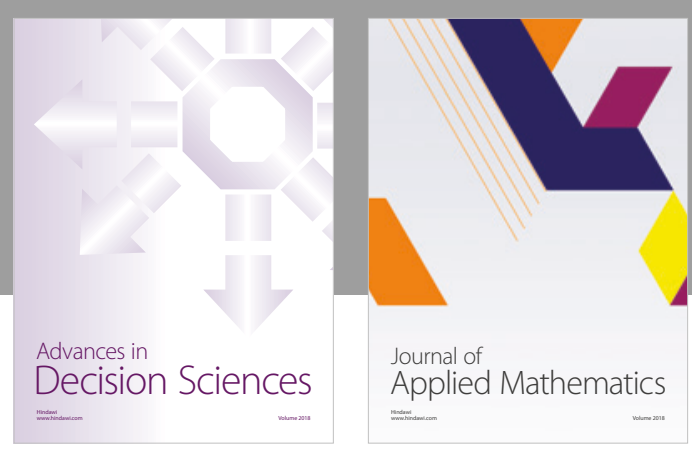

Journal of

Applied Mathematics
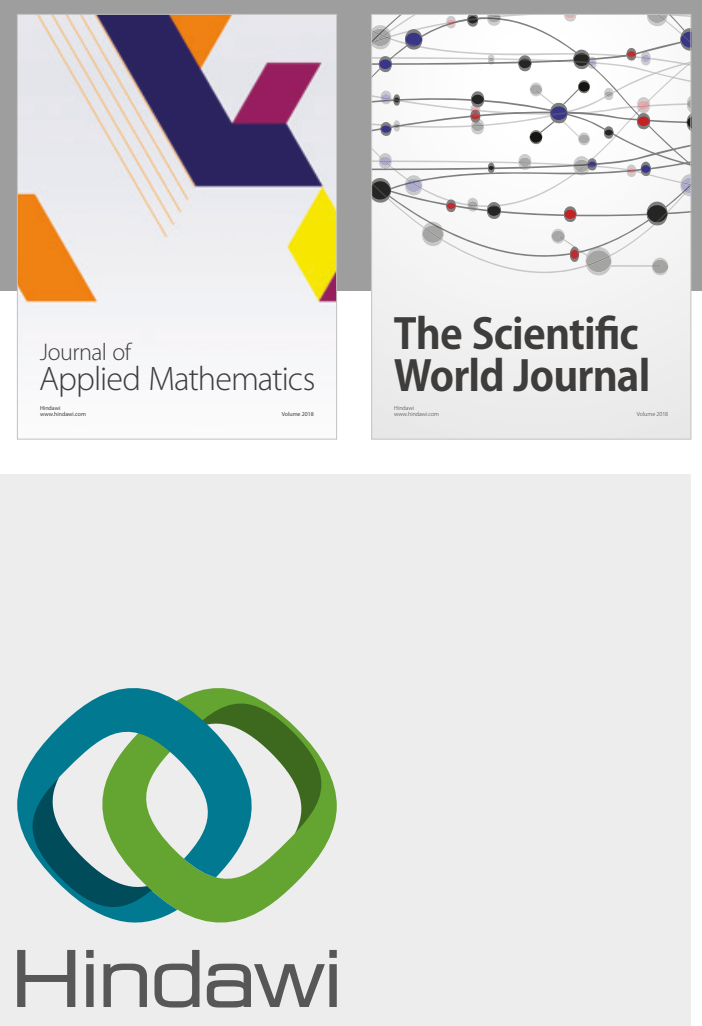

Submit your manuscripts at

www.hindawi.com

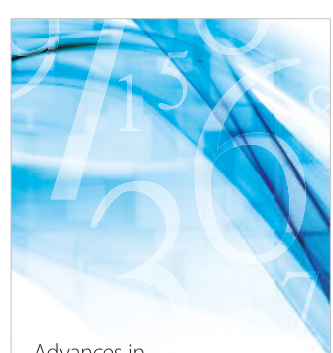

Advances in
Numerical Analysis
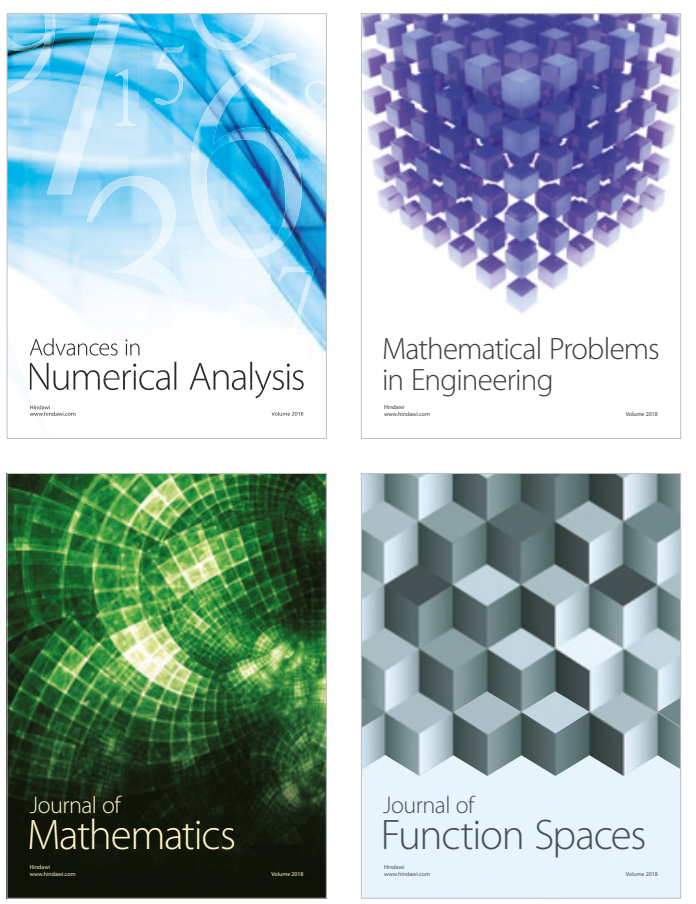

Mathematical Problems in Engineering

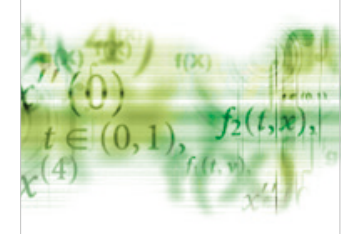

International Journal of

Differential Equations

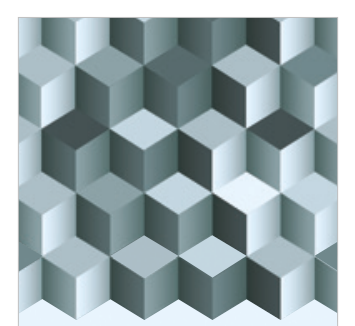

Journal of

Function Spaces

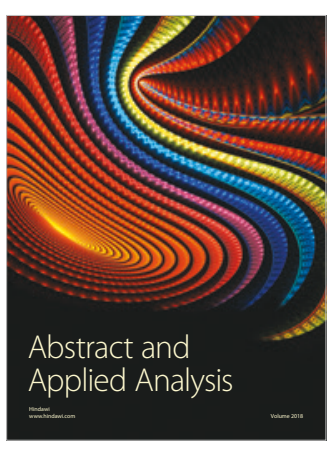

The Scientific

World Journal

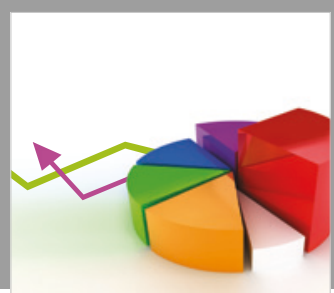

Journal of

Probability and Statistics
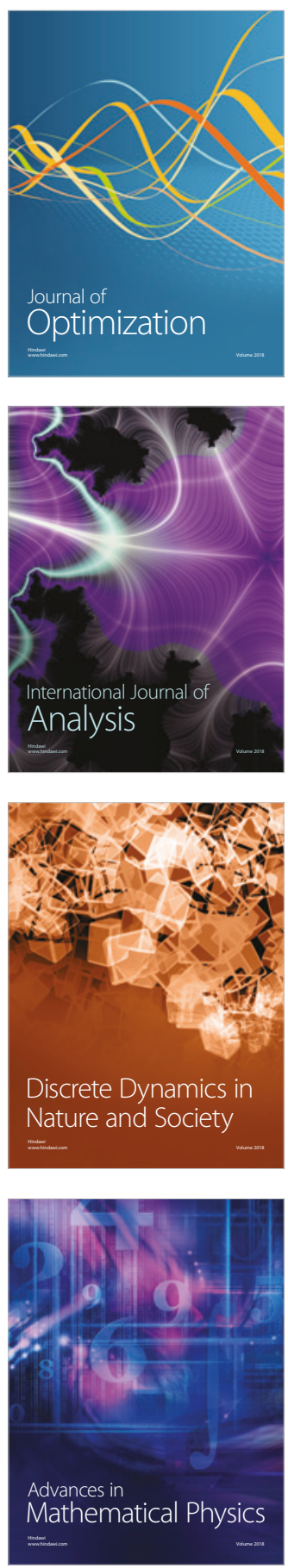